Editorial

\title{
Redox-Activated Signal Transduction Pathways Mediating Cellular Functions in Inflammation, Differentiation, Degeneration, Transformation, and Death
}

\author{
Tetsuro Kamiya, ${ }^{1}$ Michael Courtney, ${ }^{2}$ and Mikko O. Laukkanen ${ }^{3}$ \\ ${ }^{1}$ Laboratory of Clinical Pharmaceutics, Gifu Pharmaceutical University, 1-25-4 Daigakunishi, Gifu 501-1196, Japan \\ ${ }^{2}$ Neuronal Signalling Laboratory, Turku Centre for Biotechnology, University of Turku, Turku, Finland \\ ${ }^{3}$ IRCCS SDN, 80143 Naples, Italy \\ Correspondence should be addressed to Mikko O. Laukkanen; mlaukkanen@sdn-napoli.it
}

Received 20 October 2016; Accepted 24 October 2016

Copyright (C) 2016 Tetsuro Kamiya et al. This is an open access article distributed under the Creative Commons Attribution License, which permits unrestricted use, distribution, and reproduction in any medium, provided the original work is properly cited.

The early atmosphere of the earth contained low concentration of oxygen, which then slowly initiated to increase approximately 2.5 billion years ago after development of photosynthetic organisms and reached life-supporting levels 500-1000 million years ago. According to the oxygenic theory of evolution even the most primitive organisms used oxidative-antioxidative mechanisms in energy production, hence underlining the importance of reactive oxygen species (ROS) in normal cellular functions [1]. In reduction-oxidation (redox) biology the balance of oxidants and antioxidants determines the normal cellular homeostasis that is altered in tissue injuries and pathological conditions. Therefore, ROS, or unbalanced production of ROS, are often a consequence of injury or pathological condition with corresponding impact on cellular signal transduction and damage to macromolecules. The focus of this special issue is to characterize signaling pathways involved in tissue pathogenesis. Such studies potentially result in drug targets that supplement targets observed in currently used screenings [2].

ROS, especially hydrogen peroxide $\left(\mathrm{H}_{2} \mathrm{O}_{2}\right)$ and superoxide anion $\left(\mathrm{O}_{2}{ }^{--}\right)$, are important second messengers able to activate or inhibit cellular signaling. It is noteworthy that the cellular response to ROS directly correlates to their concentration: low concentration often supports growth and survival, whereas high concentration is involved in aberrant cellular behavior, such as growth arrest and transformation. Ras oncogene induced proliferative burst of primary cells, consequent cancer barrier forming senescence of the cells, and escape from premature senescence through immortalization and transformation of primary cells is a classical example of ROS involvement in aberrant cellular functions [3]. Epigenetics, a topic discussed in the special issue by T. Kamiya et al., is essentially involved in carcinogenesis. The role of ROS in epigenetic regulation of gene expression through DNA methylation, histone methylation, and histone acetylation has received attention in the research society emphasizing the significant liaison of ROS with carcinogenesis. Part of the epigenetic control in carcinogenesis is derived from tumor stroma cells. Tumor stroma develops in several phases responding to the needs of the epithelial cancer cells. Thus, the continuously growing tumor contains all developmental phases of stroma [4]. Mesenchymal stem cells (MSC) are a rich source of ROS (reviewed in the current issue by R. A. Denu et al.) being able to regulate tumor cells growth and stromal inflammatory cell immunomodulatory properties by, for example, affecting macrophage maturation.

Infections and inflammation are frequently connected to aberrant ROS production. In the current issue D. Limongi and S. Baldelli review the role of infections in neurogenerative diseases. Increased ROS levels with consequent increased inflammatory cytokine expression via activation of Toll like receptor 4 (TLR4) signaling have been suggested to sensitize cells to amyotrophic lateral sclerosis (ALS) development in patients who carry mutations in cupper zinc superoxide 
dismutase (SOD1) gene in astrocytes and in microglia. Wild type SOD1 binds small GTPase RAC1 subunit of NADPH oxidase complex in a redox sensitive manner responding to increasing concentration of $\mathrm{H}_{2} \mathrm{O}_{2}$ by disassociating from RAC1. Mutant SOD1 lacks the redox sensitivity in RAC1 binding, therefore causing continuous accumulation of $\mathrm{H}_{2} \mathrm{O}_{2}$, which then stimulates TLR4 signal transduction and downstream inflammatory cytokine expression. Although the function of inflammatory cytokines in ALS pathogenesis is not completely understood, both increased ROS and inflammatory cytokine concentrations have been demonstrated to be risk factors in ALS development. Extracellular superoxide dismutase (SOD3) previously characterized in atherosclerotic diseases [5] has been shown to attenuate nonbacterial inflammation, mainly macrophage migration, in ischemic injuries by reducing inflammatory cytokine and adhesion molecule expression [6]. Hence, these data demonstrate that dismutase reaction by SOD1 and SOD3, a catalysis of $\mathrm{O}_{2}{ }^{\cdot-}$ to $\mathrm{H}_{2} \mathrm{O}_{2}$, has an opposite effect on inflammatory cytokine expression correlating to cellular location of the SOD enzymes.

Although the role of ROS and redox genes is well characterized in several pathological conditions redox balance is a sum of various actions in cellular homeostasis, therefore being challenging to manipulate. Recently a number of studies have pointed out a single transcription factor in redox system, NF-E2-related factor-2 (NRF2), which coordinates oxidant-antioxidant balance in collaboration with Keap1 (reviewed in the current issue by D. I. Kim et al. and A. Sparaneo et al.). Although these factors are attractive drug targets as they are involved in the development of severe pathological conditions, such as malignant cancer cell chemoresistance, the overall development of antioxidative drugs has faced several obstacles. A number of antioxidative molecules tested in cell models in vitro, in preclinical models in vivo, and in clinical trials have demonstrated the diversity of redox balance, even suggesting unexpected negative outcomes. N-Acetyl-L-cystein (NAC), a precursor of glutathione, represents one of the most thoroughly investigated molecules, which has been tested in several clinical trials related to oxidative stress derived diseases, such as chronic inflammation, brain injury, lung diseases, acute liver diseases, cardiovascular diseases, and fibrotic diseases. NAC primarily neutralizes $\mathrm{O}_{2}{ }^{\bullet-}$, although it has been reported to reduce $\mathrm{H}_{2} \mathrm{O}_{2}$ concentrations and therefore recognized as an antioxidative molecule. Although NAC potentially attenuates the oxidative damage in cardiovascular and liver injuries in short-term treatment, it has less prominent effect on long-term survival of liver injury patients [7, 8]. A more sophisticated approach in the control of redox balance is to focus on signaling routes that mediate the effect of ROS in tissue injuries or cancer, as suggested by P. A. N. Thi et al. in the current issue article, which reports the use of MEK inhibitor (PD98059) to protect brain and cardiac tissue in cardiac arrest remote conditioning model.

Despite impressive resources utilized in characterization of ROS, their function, and manipulation of production in disease progression, we still do not quite understand the connection between ROS production and cellular signal transduction. Further analysis in characterization of ROS regulated signaling routes could reveal critical molecules needed for ROS production, such as small GTPase RAC1 that is involved in NADPH oxidase function and in SOD3 signaling $[9,10]$.

\section{Tetsuro Kamiya \\ Michael Courtney Mikko O. Laukkanen}

\section{References}

[1] D. E. Canfield and A. Teske, "Late proterozoic rise in atmospheric oxygen concentration inferred from phylogenetic and sulphur-isotope studies," Nature, vol. 382, no. 6587, pp. 127-132, 1996.

[2] M. C. Cantisani, A. Parascandolo, M. Perälä et al., "A loss-offunction genetic screening identifies novel mediators of thyroid cancer cell viability," Oncotarget, vol. 7, no. 19, pp. 28510-28522, 2016.

[3] C. J. Sarkisian, B. A. Keister, D. B. Stairs, R. B. Boxer, S. E. Moody, and L. A. Chodosh, "Dose-dependent oncogeneinduced senescence in vivo and its evasion during mammary tumorigenesis," Nature Cell Biology, vol. 9, no. 5, pp. 493-505, 2007.

[4] F. Cammarota and M. O. Laukkanen, "Mesenchymal stem/ stromal cells in stromal evolution and cancer progression," Stem Cells International, vol. 2016, Article ID 4824573, 11 pages, 2016.

[5] M. O. Laukkanen, P. Leppänen, P. Turunen, E. PorkkalaSarataho, J. T. Salonen, and S. Ylä-Herttuala, "Gene transfer of extracellular superoxide dismutase to atherosclerotic mice," Antioxidants and Redox Signaling, vol. 3, no. 3, pp. 397-402, 2001.

[6] J. P. Laurila, L. E. Laatikainen, M. D. Castellone, and M. O. Laukkanen, "SOD3 reduces inflammatory cell migration by regulating adhesion molecule and cytokine expression," PLoS ONE, vol. 4, no. 6, Article ID e5786, 2009.

[7] M. Ozaydin, O. Peker, D. Erdogan et al., "Oxidative status, inflammation, and postoperative atrial fibrillation with metoprolol vs carvedilol or carvedilol plus n-acetyl cysteine treatment," Clinical Cardiology, vol. 37, no. 5, pp. 300-306, 2014.

[8] E. Nguyen-Khac, T. Thevenot, M.-A. Piquet et al., "Glucocorticoids plus N-Acetylcysteine in severe alcoholic hepatitis," New England Journal of Medicine, vol. 365, no. 19, pp. 1781-1789, 2011.

[9] M. M. Harraz, J. J. Marden, W. Zhou et al., "SOD1 mutations disrupt redox-sensitive Rac regulation of NADPH oxidase in a familial ALS model," The Journal of Clinical Investigation, vol. 118, no. 2, pp. 659-670, 2008.

[10] M. O. Laukkanen, F. Cammarota, T. Esposito, M. Salvatore, and M. D. Castellone, "Extracellular superoxide dismutase regulates the expression of small GTPase regulatory proteins GEFs, GAPs, and GDI," PLoS ONE, vol. 10, no. 3, Article ID e0121441, 2015. 


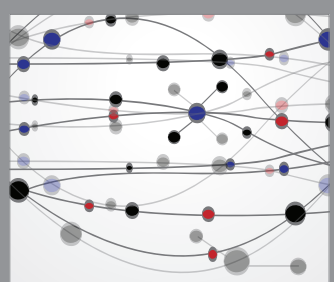

The Scientific World Journal
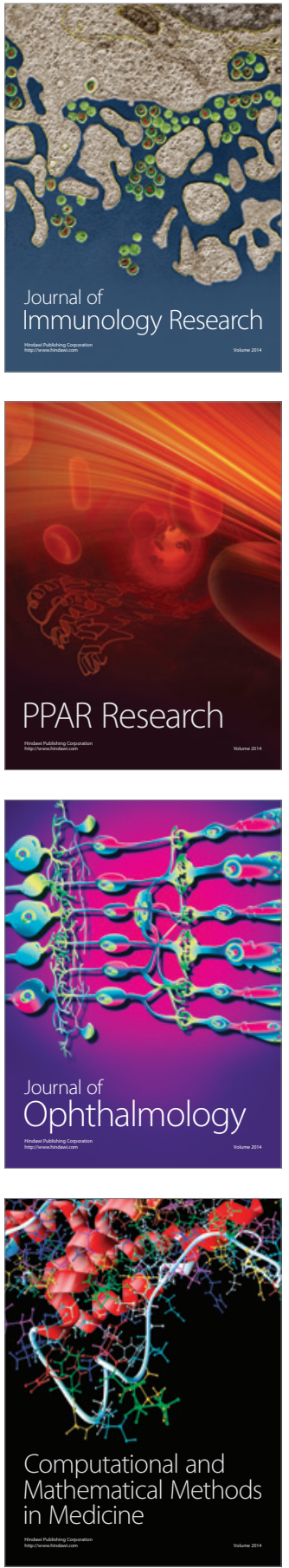

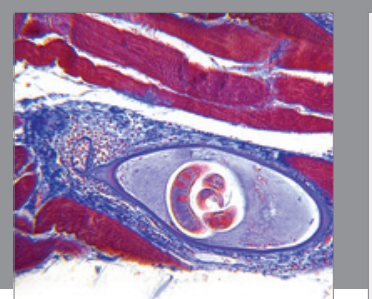

Gastroenterology Research and Practice

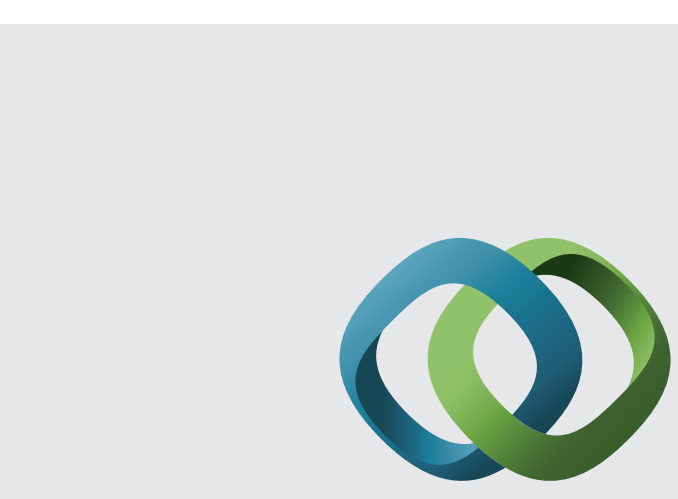

\section{Hindawi}

Submit your manuscripts at

http://www.hindawi.com
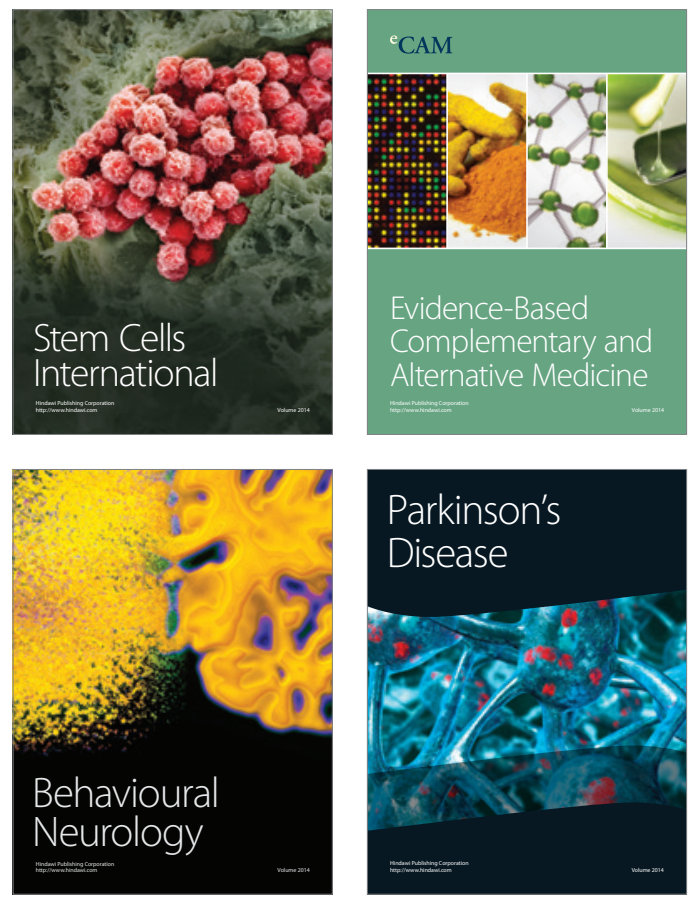
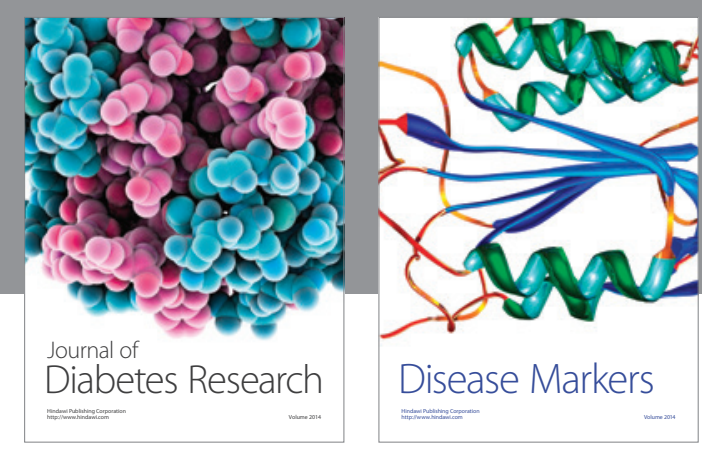

Disease Markers
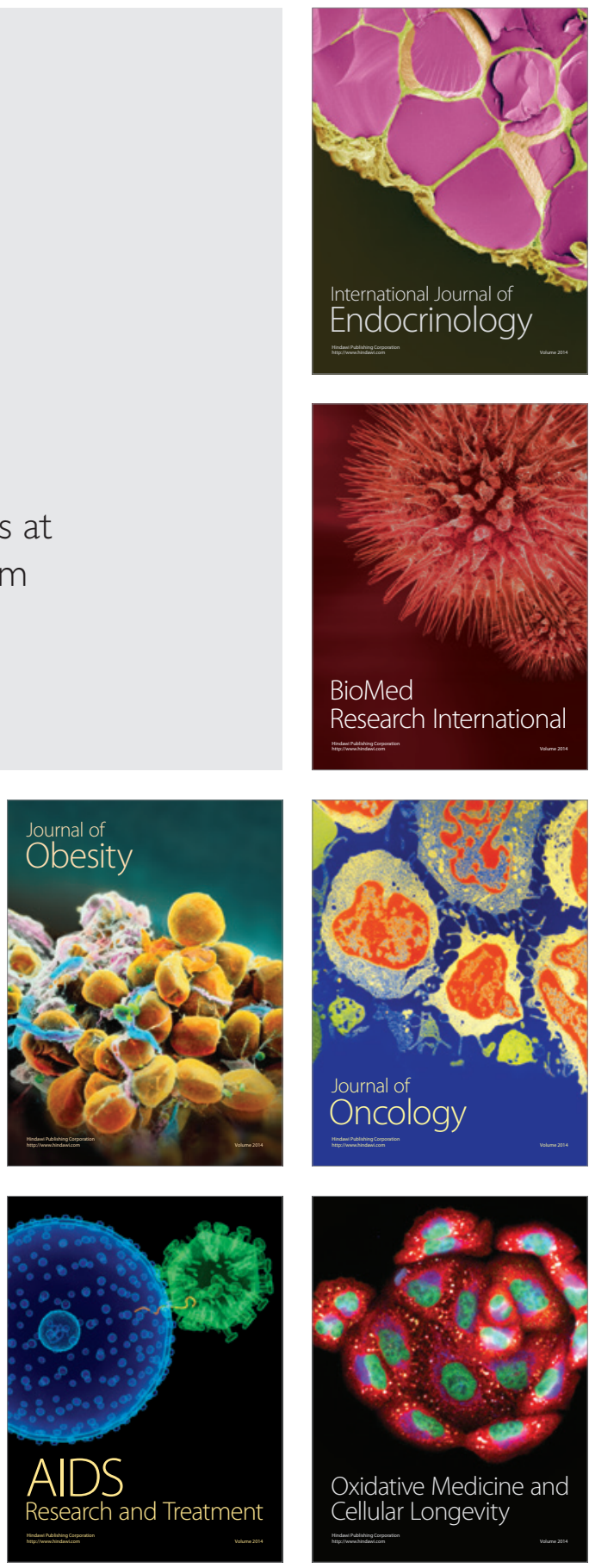